MATEC Web of Conferences 4, 01001 (2013)

DOI: $10.1051 /$ matecconf/20130401001

(c) Owned by the authors, published by EDP Sciences, 2013

\title{
Fabrication of fluorescent chitosan-containing microcapsules
}

\author{
R. Zhang ${ }^{1,2,3^{*}}$, J. Xin ${ }^{3}$, and H. Möhwald ${ }^{4}$
}

${ }^{1}$ Key Laboratory of Special Functional Aggregated Materials of the Ministry of Education, Shandong University, Jinan 250100, P. R. China

${ }^{2}$ National Engineering Technology Research Center for Colloidal Materials, Shandong University, Jinan 250100, P. R. China

${ }^{3}$ Key Laboratory of Colloid and Interface Chemistry of the Ministry of Education, Shandong University, Jinan 250100, P. R. China

${ }^{4}$ Department of Interfaces, Max Planck Institute of Colloids and Interfaces, Potsdam14424, Germany.

\begin{abstract}
Intense emission peaks of $\mathrm{Eu}(\mathrm{DBM})_{3}$ Phen (DBM and Phen are dibenzoylmethane and 1,10phenanthroline, respectively) in the microcapsules containing molecules of quaternary ammonium chitosan (QACS) and sodium alginate are observed. The microcapsules are assembled by using $\mathrm{CaCO}_{3}$ particles as template cores by the layer-by-layer (LbL) technique. Observation of microcapsules by the fluorescence mode and the transmission mode in the confocal laser scanning microscopy shows that the microcapsules are intact after core decomposition. Fluorescence under ultraviolet irradiation comes directly from the $\mathrm{Eu}(\mathrm{DBM})_{3} \mathrm{Phen}$. Homogeneous assembly of $\mathrm{Eu}(\mathrm{DBM})_{3}$ Phen can be deduced due to the homogeneous fluorescence of the microcapsules in the fluorescence micrographs. The microcapsules show adherence to solid substrates due to large quantities of hydroxyl groups of QACS. AFM measurements of dried hollow microcapsules with only 4 bilayers of $(\mathrm{CS} / \mathrm{SA})$ fabricated with $\mathrm{Eu}(\mathrm{DBM})_{3}$ Phen show the intact shell with a thickness of $3.0 \mathrm{~nm}$. Regarding the biocompatible natural polysaccharides and the intense fluorescence emission, the microcapsules in this work might be of great importance in potential application in drug delivery and bioassay.
\end{abstract}

\section{Introduction}

Chitosan is naturally biodegradable, characterizing in no accumulation or cytotoxicity in biological organisms [1]. So it has been extensively studied as a shell material not only in capsules whose diameter is to several hundreds of micrometers and thickness to tens of nanometers per deposited layer [2-3], and in microcapsules whose diameter and thickness of each layer are about several micrometers and $1 \mathrm{~nm}$ [4], respectively.

The layer-by-layer (LbL) technique [5-8] has been well developed not only to assemble two-dimensional (2D) planar films on solid substrates, but also to threedimensional (3D) capsules by replacing the planar solid substrates with colloidal particles. After core decomposition by appropriate solvents corresponding to organic or inorganic cores, hollow microcapsules can be obtained. Microcapsules of synthetic polyelectrolytes have many superior properties such as high Young's modulus, perfect stability, and switchable channels in shells, which is basically important in the practical applications rather than pure researches of physical chemistry and self-assembly.
Europium $\beta$-diketone complexes have sharp emission peaks of the Eu(III) ions with narrow peak width at half-height. Such unusual spectroscopic character enables the complexes to serve as molecular probes in bioassay [9-10] for less peak overlap and high image resolution. Fluorescence mechanisms of the efficient energy transfer from the $\beta$-diketone ligands as energy-harvesting antenna to the central $\mathrm{Eu}(\mathrm{III})$ ions [11-12] have been revealed, and Eu(III)-containing microcapsules have been LbL self-assembled with the shells of synthetic polyelectrolytes like poly(sodium 4styrenesulfonate) (PSS) and poly(allylamine hydrochloride) (PAH) [13-14]. However, the fabrication of $\mathrm{Eu}(\mathrm{III}) \quad \beta$-diketone complexes in the microcapsule shell of natural biomolecules like polysaccharides have not been reported.

$\mathrm{Eu}(\mathrm{DBM})_{3}$ Phen (DBM and Phen denote dibenzoylmethane and 1,10-phenanthroline, respectively) is one $\mathrm{Eu}(\mathrm{III}) \beta$-diketone complex, in which the DBM is a $\beta$-diketone ligand, chelating the $\mathrm{Eu}(\mathrm{III})$ together with the Phen ligand to form the complex, $\mathrm{Eu}(\mathrm{DBM})_{3}$ Phen. In this work, we assemble the $\mathrm{Eu}(\mathrm{DBM})_{3}$ Phen and the polysaccharides 
(quaternary ammonium chitosan, QACS, and sodium alginate, SA) molecules on colloidal $\mathrm{CaCO}_{3}$ particles as the template core by the $\mathrm{LbL}$ technique to obtain microcapsules. The fluorescence and the morphology of the microcapsules are addressed.

\section{Materials and Methods}

2.1 Materials SA was from Sigma and used without further treatment. $\mathrm{Eu}(\mathrm{DBM})_{3}$ Phen was synthesized according to literature methods [15-16]. QACS was synthesized according to literature methods [17-19]. Figure 1 shows the schematic molecular structure of $\mathrm{Eu}(\mathrm{DBM})_{3}$ Phen and QACS. The viscosity and degree of deacetylation of QACS were $200-800 \mathrm{cP}(1 \%$ in $1 \%$ acetic acid) and 75-85\%, respectively. $\mathrm{CaCl}_{2}, \mathrm{Na}_{2} \mathrm{CO}_{3}$, $\mathrm{NaCl}, \mathrm{NaOH}, \mathrm{NaCl}$, acetic acid and disodium ethylendiaminetetraacetic acid (EDTA) were all analytical reagents and used without further purification. $\mathrm{CaCO}_{3}$ microparticles were prepared according to literature methods [20]. The solutions of QACS (1.0 $\mathrm{mg} / \mathrm{mL}$ in $0.3 \mathrm{M} \mathrm{NaCl}$ and $0.02 \mathrm{M}$ acetic acid) and SA $(1.0 \mathrm{mg} / \mathrm{mL}$ in $0.3 \mathrm{M} \mathrm{NaCl})$ were left under stirring at room temperature overnight, adjusted to $\mathrm{pH} 5.0$ and filtered through membranes with pores of $8.0 \mu \mathrm{m}$ in diameter before the LbL assembly. Water used in all experiments had a resistivity higher than $18.2 \mathrm{M} \Omega \cdot \mathrm{cm}$.
A

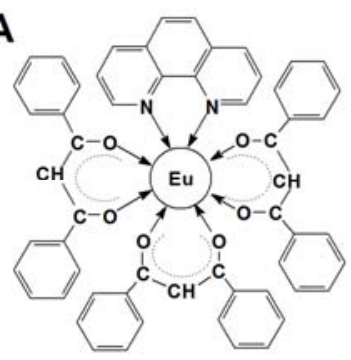

B

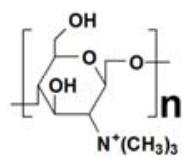

Fig. 1. Schematic molecular structure of $(\mathrm{A}) \mathrm{Eu}(\mathrm{DBM})_{3}$ Phen and (B) QACS

\subsection{Fabrication of fluorescent microcapsules} Microcapsules were fabricated first by incubation of $\mathrm{CaCO}_{3}$ microparticles for $20 \mathrm{~min}$ alternatingly in solutions of QACS and SA for 4 times. The dispersion solutions were centrifuged $(1500 \mathrm{rpm}, 5 \mathrm{~min})$ to remove the supernatant and washed for 3 times ( 5 min each) with water. Gentle sonication for $10 \mathrm{~s}$ was applied at the beginning of deposition and washing to separate aggregated particles and to promote homogeneous deposition of the polysaccharides onto well separated microparticles. Then, a 0.1 M EDTA solution was used to dissolve the $\mathrm{CaCO}_{3}$ template core and so hollow (QACS/SA) $)_{4}$ microcapsules were obtained, followed by washing for 3 times with centrifugation (3500 rpm, $1 \mathrm{~h}$ ). Finally, hollow (QACS/SA) $)_{4}$ microcapsules were incubated in the saturated ethanol solution of $\mathrm{Eu}(\mathrm{DBM})_{3}$ Phen for $20 \mathrm{~min}$ and followed by washing for 3 times with centrifugation $(3500 \mathrm{rpm}, 1 \mathrm{~h})$ with pure water.

\subsection{Characterization of the microcapsules}

Fluorescence spectra were recorded on an Edinburgh FLS920 (UK) spectrophotometer. An Olympus IX 81 microscope (Japan) was used to observe the morphologies of fluorescent microcapsules, irradiated by a mercury lamp for the fluorescence images. An excitation filter allowed the beam with the wavelength range of 330-385 $\mathrm{nm}$ to irradiate the microcapsules. The transmission images were obtained under lumination of the Ar laser. A 100x oil immersion objective and a DP70 CCD camera were used. Atomic force microscopy (AFM) images were taken on Digital Instrument Nanoscope IIIa with the tapping mode (USA) at a scan rate of $1 \mathrm{~Hz}$.

\section{Results and Discussion}

Figure 2 shows the fluorescence emission spectrum of $\mathrm{Eu}(\mathrm{DBM})_{3}$ Phen in the $\left[(\mathrm{QACS} / \mathrm{SA})_{4} \mathrm{Eu}(\mathrm{DBM})_{3}\right.$ Phen $]$ microcapsules. Three main emission peaks are at 549 , 585.5 and $613.5 \mathrm{~nm}$. The maximum fluorescence intensity at $613.5 \mathrm{~nm}$ is about 670 , with the width at half-maximum intensity (335) of 618.3-611.1 = 7.2 nm. Such a narrow width demonstrates the characteristic emission of $\mathrm{Eu}(\mathrm{III})$, which is not influenced by polysaccharide molecules. The width is much narrower compared to a value larger than $30 \mathrm{~nm}$, the general width at half-maximum intensity of emission of green fluorescence protein dyes. This is of paramount importance in multicolor assay, since overlay of emission peaks would seriously lower the imaging resolution.

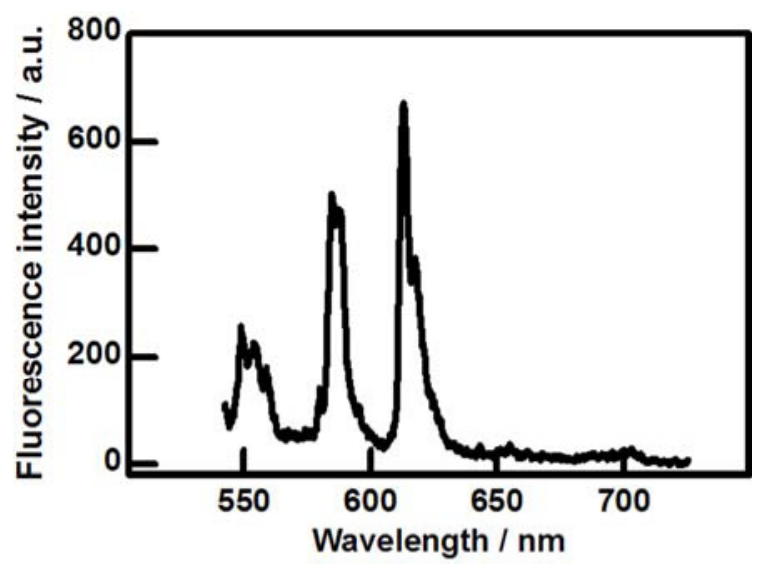

Fig. 2. Fluorescence emission spectrum of $\left[(\mathrm{QACS} / \mathrm{SA})_{4} \mathrm{Eu}(\mathrm{DBM})_{3}\right.$ Phen] microcapsules

The hollow microcapsules after dissolution of the $\mathrm{CaCO}_{3}$ core emit so intense fluorescence that brilliant red light can be observed by bare eyes, when irradiated by ultraviolet excitation light. Under microscope, the microcapsules appear as rings. The morphology of $\left[(\mathrm{QACS} / \mathrm{SA})_{4} \mathrm{Eu}(\mathrm{DBM})_{3} \mathrm{Phen}\right]$ microcapsules in water is shown in Figure 3. The microcapsules are intact and spherical, which is attributed to that the surface tension reducing the membrane surface is balanced by electrostatic repulsion between excess charges in 
polysaccharides molecules. As a result, electrostatic repulsion maintains the morphology of the shell containing polysaccharides and $\mathrm{Eu}(\mathrm{DBM})_{3}$ Phen. It's worthy to note that the QACS in this work is a derivative of chitosan (CS), the latter is modified with a positively charged $-\mathrm{N}\left(\mathrm{CH}_{3}\right)_{3}{ }^{+}$group to increase the solubility in water and to facilitate the electrostatic $\mathrm{LbL}$ assembly. A general $\mathrm{CS}$ is poorly insoluble in water.

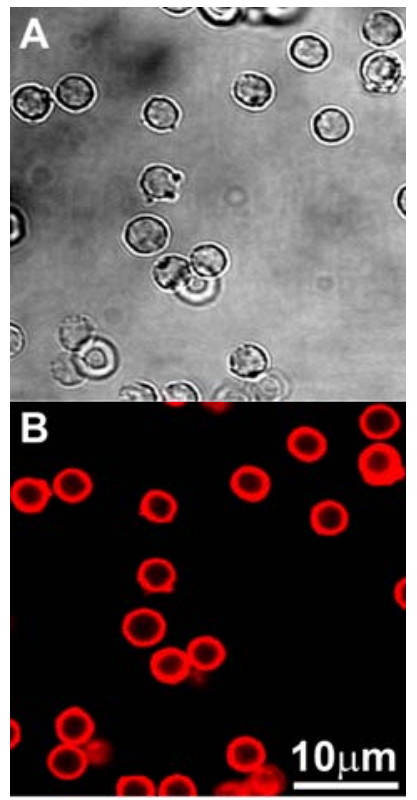

Fig. 3. Micrographs of fluorescent $\left[(\mathrm{QACS} / \mathrm{SA})_{4} \mathrm{Eu}(\mathrm{DBM})_{3}\right.$ Phen] microcapsules. A: transmission mode; $B$ : fluorescence mode

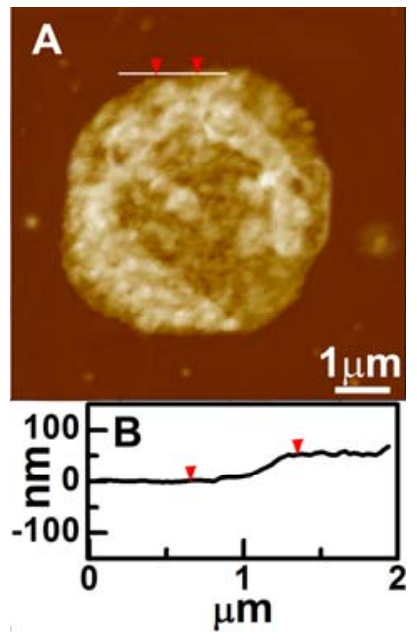

Fig. 4. (A) AFM height image of a hollow $\left[(\mathrm{QACS} / \mathrm{SA})_{4} \mathrm{Eu}(\mathrm{DBM})_{3}\right.$ Phen] microcapsule, $(\mathrm{B})$ section analysis of the line drawn in (A)

The microcapsules emit homogeneous fluorescence. So on one hand, the assembly of $\operatorname{Eu}(\mathrm{DBM})_{3}$ Phen in the polysaccharides shell matrix is shown to be well achieved. On the other hand, the $\mathrm{Eu}(\mathrm{DBM})_{3}$ Phen is shown to distribute homogeneously in the shell. The shell of microcapsules is brighter than the center core, which not only facilitates the analysis of the diameter of the microcapsule, but also indicates the interaction between the $\mathrm{Eu}(\mathrm{DBM})_{3}$ Phen and the polysaccharide molecules. The diameter is analyzed to be 3.5-5.0 $\mu \mathrm{m}$, nearly the same as that of the $\mathrm{CaCO}_{3}$ template cores. Both the $\operatorname{Eu}(\mathrm{DBM})_{3}$ Phen and the molecular main chains of polysaccharide are hydrophobic. So due to the hydrophobic-hydrophobic interaction, the $\mathrm{Eu}(\mathrm{DBM})_{3}$ Phen preferably deposits on the main chain, leading to the brighter shell formed by closely assembled QACS and SA molecules. This kind of $\mathrm{Eu}(\mathrm{DBM})_{3}$ Phen-polysaccharide interaction also contributes to the intense fluorescence emission, since the hydrophobic main chains of polysaccharide repel water molecules, energy loss through the non-radiative deactivation from the ${ }^{5} \mathrm{D}_{0}$ excitation state of the $\mathrm{Eu}(\mathrm{III})$ to the high-energy $\mathrm{O}-\mathrm{H}$ vibration is effectively decreased [21-22].

The $\left[(\mathrm{QACS} / \mathrm{SA})_{4} \mathrm{Eu}(\mathrm{DBM})_{3}\right.$ Phen $]$ microcapsules show adherence to solid substrates, mostly stably sitting on solid substrates against rinsing with water. The adhesive force can be due to large quantities of hydroxyl groups in QACS, which might form hydrogen bonds with hydroxyl groups on solid substrates.

The LbL technique enables the fabrication of microcapsules with each layer of about $1 \mathrm{~nm}$ in thickness, making the atomic force microscopy (AFM) a mostly-used tool to study microcapsules [14]. The surface morphology and shell thickness of $\mathrm{Eu}(\mathrm{DBM})_{3}$ Phen-containing polysaccharide microcapsules was so studied by AFM in tapping mode. Figure 4A shows a typical AFM height image of a dried hollow [(QACS/SA $)_{4} \mathrm{Eu}(\mathrm{DBM})_{3}$ Phen $]$ microcapsule. Continuous and intact shell is observed. The dried microcapsules exhibit folds and creases, due to the evaporation of the water molecules [23]. The intact dried hollow capsule is similar to those from synthetic polyelectrolytes, further confirming that the QACS and the SA are good shell material candidates for fabricating mechanically stable microcapsules, even mixed with the fluorescent $\mathrm{Eu}(\mathrm{DBM})_{3}$ Phen. This is of paramount importance in potential applications as bioassay probes and drug carriers. Study on the toxicity, stability and release profile of the microcapsules is ongoing. Each layer of polysaccharide or $\mathrm{Eu}(\mathrm{DBM})_{3}$ Phen in the $\left[(\mathrm{QACS} / \mathrm{SA})_{4} \mathrm{Eu}(\mathrm{DBM})_{3} \mathrm{Phen}\right]$ microcapsules have a thickness of about $3.0 \mathrm{~nm}$ and roughness of $4.5 \mathrm{~nm}, \mathrm{ca}$. 3 times of those from synthetic polyelectrolytes [24]. (QACS/SA) 4 microcapsules also exhibited folds and creases, as shown in the AFM images of the microcapsules with $\mathrm{Eu}(\mathrm{DBM})_{3}$ Phen. In other words, there is no morphology difference on the AFM images between the microcapsules in the absence or presence of the $\mathrm{Eu}(\mathrm{DBM})_{3}$ Phen.

\section{Conclusion}

In conclusion, fluorescence microcapsules of polysaccharides with $\mathrm{Eu}(\mathrm{DBM})_{3}$ Phen are assembled 
using the $\mathrm{LbL}$ technique, intact both in aqueous solution and after air-dried. The capsules are spherical in solution and show folds and creases after air-dried. Regarding the intense red fluorescence emission under ultraviolet excitation and the natural polysaccharide shell materials, the microcapsules in this work might benefit in potential applications in bioassay and drug delivery.

\section{Acknowledgements}

We thank the National Nature Science Foundation of China (No. 21273135), Shandong Provincial Natural Science Foundation, China (ZR2010BM039), Independent Innovation Foundation of Shandong University (2011JC026), and the Max Planck Society, Germany.

\section{References}

1. H. Onishi, Y. Machida, Biomaterials, 20, 175 (1999)

2. H.G. Xie, X.X. Li, G.J. Lv, W.Y. Xie, J. Zhu, T. Luxbacher, R. Ma, X.J. Ma, J. Biomed. Mater. Res., Part A, 92A, 1357 (2010)

3. Q.S. Zhao, Q.X. Ji, X.J. Cheng, G.Z. Sun, C. Ran, B. Zhao, X.G. Chen, J. Sol-Gel. Sci. Technol., 54, 232 (2010)

4. Q.H. Zhao, B.S. Han, Z.H. Wang, C.Y. Gao, C.H. Peng, J.C. Shen, Nanomedicine, 3, 63 (2007)

5. E. Donath, G.B. Sukhorukov, F. Caruso, S.A. Davis, H. Möhwald, Angew. Chem. Int. Ed., 37, 2202 (1998)

6. G.B. Sukhorukov, E. Donath, S. Davis, H. Lichtenfeld, F. Caruso, V.I. Popov, H. Möhwald, Polym. Adv. Technol., 9, 759 (1998)

7. Y. Wang, A.S. Angelatos, F. Caruso, Chem. Mater., 20, 848 (2008)

8. H.L. Tang, C.H. Yu, W. Oduoro, H.Y. He, S.C. Tsang, Langmuir, 24, 1587 (2008)

9. V. Bekiari, P. Lianos, Adv. Mater., 10, 1455 (1998)

10. Y. Bruemmel, C.P.y. Chan, R. Renneberg, A. Thuenemann, M. Seydack, Langmuir, 20, 9371 (2004)

11. R.J. Zhang, K.Z. Yang, Langmuir, 13, 7141 (1997)

12. P. Andrew, W.L. Barnes, Science, 290, 785 (2000)

13. D.G. Shchukin, G.B. Sukhorukov, H. Möhwald, J. Phys. Chem. B, 108, 19109 (2004)

14. R.J. Zhang, J.W. Cui, D.M. Lu, W.G. Hou, Chem. Commun., 1547 (2007)

15. H. Bauer, J. Blanc, D.L. Ross, J. Am. Chem. Soc., 86, 5125 (1964)

16. L.R. Melby, N.J. Rose, E. Abramson, J.C. Caris, J. Am. Chem. Soc., 86, 5117 (1964)

17. X. Chen, Y. Yuan, L. Zhang, X. Hong, Chin. J. Synth. Chem., 12, 85 (2004)

18. M. Thanou, B.I. Florea, M. Geldof, H.E. Junginger, G. Borchard, Biomaterials, 23, 153 (2002)
19. T. Kean, S. Roth, M. Thanou, J. Controlled Release, 103, 643 (2005)

20. O. Kreft, A.M. Javier, G.B. Sukhorukov, W.J. Parak, J. Mater. Chem., 17, 4471 (2007)

21. D. Sendor, U. Kynast, Adv. Mater., 14, 1570 (2002)

22. C. Wang, R. Zhang, H. Möhwald, Langmuir, 26, 11987 (2010)

23. S. Leporatti, A. Voigt, R. Mitlöhner, G. Sukhorukov, E. Donath, H. Möhwald, Langmuir, 16, 4059 (2000)

24. Z. An, H. Möhwald, J. Li, Biomacromolecules, 7, $580(2006)$ 